\title{
Generating relations and other results associated with some families of the extended Hurwitz-Lerch Zeta functions
}

\author{
Hari M Srivastava
}

\begin{abstract}
Motivated essentially by recent works by several authors (see, for example, Bin-Saad [Math J Okayama Univ 49:37-52, 2007] and Katsurada [Publ Inst Math (Beograd) (Nouvelle Ser) 62(76):13-25, 1997], the main objective in this paper is to present a systematic investigation of numerous interesting properties of some families of generating functions and their partial sums which are associated with various classes of the extended Hurwitz-Lerch Zeta functions. Our main results would generalize and extend the aforementioned recent work by Bin-Saad [Math J Okayama Univ 49:37-52, 2007] (see also Katsurada [Publ Inst Math (Beograd) (Nouvelle Ser) 62(76):13-25, 1997]). We also show the hitherto unnoticed fact that the so-called $\tau$-generalized Riemann Zeta function, which happens to be the main subject of investigation by Gupta and Kumari [Jñānābha 41:63-68, 2011]) and Saxena et al. [J Indian Acad Math 33:309-320, 2011], is simply a seemingly trivial notational variation of the familiar general Hurwitz-Lerch Zeta function $\Phi(z, s, a)$. Finally, we present a sum-integral representation formula for the general family of the extended Hurwitz-Lerch Zeta functions.

\section{Mathematics Subject Classification}

Primary 11M25, 33C60; Secondary 33C05

Keywords: Riemann, Hurwitz (or generalized) and Hurwitz-Lerch Zeta functions, Lerch Zeta function and the Polylogarithmic (or de Jonquière's) function, General Hurwitz-Lerch Zeta function, Gauss and Kummer hypergeometric functions, Fox-Wright $\Psi$-function and the $\bar{H}$-function; Mittag-Leffler type functions, Mellin-Barnes type integral representations and Meromorphic continuation, Generating functions and Eulerian Gamma-function and Beta-function integral representations
\end{abstract}

\section{Introduction and preliminaries}

Throughout our present investigation, we use the following standard notations:

$$
\mathbb{N}:=\{1,2,3, \cdots\}, \quad \mathbb{N}_{0}:=\{0,1,2,3, \cdots\}=\mathbb{N} \cup\{0\}
$$

and

$$
\mathbb{Z}^{-}:=\{-1,-2,-3, \cdots\}=\mathbb{Z}_{0}^{-} \backslash\{0\} .
$$

Here, as usual, $\mathbb{Z}$ denotes the set of integers, $\mathbb{R}$ denotes the set of real numbers, $\mathbb{R}^{+}$denotes the set of positive real numbers and $\mathbb{C}$ denotes the set of complex numbers.

The familiar general Hurwitz-Lerch Zeta function $\Phi(z, s, a)$ defined by (see, for example, (Erdélyi et al. 1953,

Correspondence: harimsri@math.uvic.ca

Department of Mathematics and Statistics, University of Victoria, Victoria, British Columbia V8W 3R4, Canada p. 27. Eq. 1.11 (1)); see also Srivastava and Choi ((2001, p. 121 et seq.) and (Srivastava and Choi 2012), p. 194 et seq.)

$$
\Phi(z, s, a):=\sum_{n=0}^{\infty} \frac{z^{n}}{(n+a)^{s}}
$$

$\left(a \in \mathbb{C} \backslash \mathbb{Z}_{0}^{-} ; s \in \mathbb{C}\right.$ when $|z|<1 ; \Re(s)>1$ when $\left.|z|=1\right)$

contains, as its special cases, not only the Riemann Zeta function $\zeta(s)$, the Hurwitz (or generalized) Zeta function $\zeta(s, a)$ and the Lerch Zeta function $\ell_{s}(\xi)$ defined by (see, for details, (Erdélyi et al. 1953, Chapter I) and Srivastava and Choi ((2001), Chapter 2)

$$
\zeta(s):=\sum_{n=0}^{\infty} \frac{1}{(n+1)^{s}}=\Phi(1, s, 1)=\zeta(s, 1) \quad(\Re(s)>1),
$$

\section{Springer}

(c) 2013 Srivastava; licensee Springer. This is an Open Access article distributed under the terms of the Creative Commons Attribution License (http://creativecommons.org/licenses/by/2.0), which permits unrestricted use, distribution, and reproduction in any medium, provided the original work is properly cited. 
$\zeta(s, a):=\sum_{n=0}^{\infty} \frac{1}{(n+a)^{s}}=\Phi(1, s, a) \quad\left(\Re(s)>1 ; a \in \mathbb{C} \backslash \mathbb{Z}_{0}^{-}\right)$

and

$\ell_{s}(\xi):=\sum_{n=0}^{\infty} \frac{\mathrm{e}^{2 n \pi \mathrm{i} \xi}}{(n+1)^{s}}=\Phi\left(\mathrm{e}^{2 \pi \mathrm{i} \xi}, s, 1\right) \quad(\Re(s)>1 ; \xi \in \mathbb{R})$,

respectively, but also such other important functions of Analytic Number Theory as the Polylogarithmic function (or de Jonquière's function) $\mathrm{Li}_{s}(z)$ :

$$
\begin{aligned}
& \operatorname{Li}_{s}(z):=\sum_{n=1}^{\infty} \frac{z^{n}}{n^{s}}=z \Phi(z, s, 1) \\
& (s \in \mathbb{C} \quad \text { when } \quad|z|<1 ; \Re(s)>1 \quad \text { when } \quad|z|=1)
\end{aligned}
$$

and the Lipschitz-Lerch Zeta function $\phi(\xi, a, s)$ (see Srivastava and Choi ((2001), p. 122, Equation 2.5 (11))):

$$
\begin{aligned}
& \phi(\xi, s, a):=\sum_{n=0}^{\infty} \frac{\mathrm{e}^{2 n \pi \mathrm{i} \xi}}{(n+a)^{s}}=\Phi\left(\mathrm{e}^{2 \pi \mathrm{i} \xi}, s, a\right) \\
& \left(a \in \mathbb{C} \backslash \mathbb{Z}_{0}^{-} ; \Re(s)>0 \text { when } \xi \in \mathbb{R} \backslash \mathbb{Z} ; \Re(s)>1\right. \\
& \text { when } \xi \in \mathbb{Z}),
\end{aligned}
$$

which was first studied by Rudolf Lipschitz (18321903) and Matyáš Lerch (1860-1922) in connection with Dirichlet's famous theorem on primes in arithmetic progressions (see also (Srivastava 2011), Section 5). Indeed, just as its aforementioned special cases $\zeta(s)$ and $\zeta(s, a)$, the Hurwitz-Lerch Zeta function $\Phi(z, s, a)$ defined by (1.1) can be continued meromorphically to the whole complex $s$-plane, except for a simple pole at $s=1$ with its residue 1. It is also known that (Erdélyi et al. 1953, p. 27, Equation $1.11(3)$ )

$$
\begin{aligned}
& \Phi(z, s, a)=\frac{1}{\Gamma(s)} \int_{0}^{\infty} \frac{t^{s-1} \mathrm{e}^{-a t}}{1-z \mathrm{e}^{-t}} \mathrm{~d} t \\
& \quad=\frac{1}{\Gamma(s)} \int_{0}^{\infty} \frac{t^{s-1} \mathrm{e}^{-(a-1) t}}{\mathrm{e}^{t}-z} \mathrm{~d} t \\
& (\Re(a)>0 ; \Re(s)>0 \text { when }|z| \leqq 1(z \neq 1) ; \Re(s)>1 \\
& \quad \text { when } z=1) .
\end{aligned}
$$

Making use of the Pochhammer symbol (or the shifted factorial $(\lambda)_{v}(\lambda, v \in \mathbb{C})$ defined, in terms of the familiar Gamma function, by

$$
\begin{aligned}
(\lambda)_{v}: & =\frac{\Gamma(\lambda+v)}{\Gamma(\lambda)} \\
& = \begin{cases}1 & (v=0 ; \lambda \in \mathbb{C} \backslash\{0\}) \\
\lambda(\lambda+1) \cdots(\lambda+n-1) & (v=n \in \mathbb{N} ; \lambda \in \mathbb{C}),\end{cases}
\end{aligned}
$$

it being understood conventionally that $(0)_{0}:=1$ and assumed tacitly that the Gamma quotient exists, we recall each of the following well-known expansion formulas:

$$
\zeta(s, a-t)=\sum_{n=0}^{\infty} \frac{(s)_{n}}{n !} \zeta(s+n, a) t^{n} \quad(|t|<|a|)
$$

and

$$
\Phi(z, s, a-t)=\sum_{n=0}^{\infty} \frac{(s)_{n}}{n !} \Phi(z, s+n, a) t^{n} \quad(|t|<|a|) .
$$

More generally, it is not difficult to show similarly that

$$
\begin{aligned}
\sum_{n=0}^{\infty} \frac{(\lambda)_{n}}{n !} \Phi(z, s+n, a) t^{n} & =\sum_{k=0}^{\infty} \frac{z^{k}}{(k+a)^{s-\lambda}(k+a-t)^{\lambda}} \\
& =: \vartheta_{\lambda}(z, t ; s, a) \quad(|t|<|a|)
\end{aligned}
$$

which would reduce immediately to the expansion formula (1.10) in its special case when $\lambda=s$. Moreover, in the limit case when

$$
t \mapsto \frac{t}{\lambda} \quad \text { and } \quad|\lambda| \rightarrow \infty,
$$

this last result (1.11) yields

$$
\begin{aligned}
\sum_{n=0}^{\infty} \Phi(z, s+n, a) \frac{t^{n}}{n !} & =\sum_{k=0}^{\infty} \frac{z^{k}}{(k+a)^{s}} \exp \left(\frac{t}{k+a}\right) \\
& =: \varphi(z, t ; s, a) \quad(|t|<\infty) .
\end{aligned}
$$

Wilton (1922/1923) applied the expansion formula (1.9) in order to rederive Burnside's formula (Erdélyi et al. 1953, p. 48, Equation 1.18 (11)) for the sum of a series involving the Hurwitz (or generalized) Zeta function $\zeta(s, a)$. Srivastava (see, for details, Srivastava (1988a;1988b)), on the other hand, made use of such expansion formulas as (1.9) and (1.10) as well as the obvious special case of (1.9) when $a=1$ for finding the sums of various classes of series involving the Riemann Zeta function $\zeta(s)$ and the Hurwitz (or generalized) Zeta function $\zeta(s, a)$ (see also Srivastava and Choi ((2001), Chapter 3) and (Srivastava and Choi 2012), Chapter 3).

Various results for the generating functions $\vartheta_{\lambda}(z, t ; s, a)$ and $\varphi(z, t ; s, a)$, which are defined by (1.11) and (1.12), respectively, were given recently by Bin-Saad (2007, p. 46, 
Equations (5.1) to (5.4)) who also considered each of the following truncated forms of these generating functions:

$$
\begin{aligned}
& \vartheta_{\lambda}^{(0, r)}(z, t ; s, a):=\sum_{k=0}^{r} \frac{z^{k}}{(k+a)^{s-\lambda}(k+a-t)^{\lambda}} \quad\left(r \in \mathbb{N}_{0}\right), \\
& \vartheta_{\lambda}^{(r+1, \infty)}(z, t ; s, a):=\sum_{k=r+1}^{\infty} \frac{z^{k}}{(k+a)^{s-\lambda}(k+a-t)^{\lambda}} \quad\left(r \in \mathbb{N}_{0}\right), \\
& \varphi^{(0, r)}(z, t ; s, a):=\sum_{k=0}^{r} \frac{z^{k}}{(k+a)^{s}} \exp \left(\frac{t}{k+a}\right) \quad\left(r \in \mathbb{N}_{0}\right)
\end{aligned}
$$

and

$$
\varphi^{(r+1, \infty)}(z, t ; s, a):=\sum_{k=r+1}^{\infty} \frac{z^{k}}{(k+a)^{s}} \exp \left(\frac{t}{k+a}\right) \quad\left(r \in \mathbb{N}_{0}\right),
$$

so that, obviously, we have

$$
\vartheta_{\lambda}^{(0, r)}(z, t ; s, a)+\vartheta_{\lambda}^{(r+1, \infty)}(z, t ; s, a)=\vartheta_{\lambda}(z, t ; s, a)
$$

and

$$
\varphi^{(0, r)}(z, t ; s, a)+\varphi^{(r+1, \infty)}(z, t ; s, a)=\varphi(z, t ; s, a)
$$

For the Riemann Zeta function $\zeta(s)$, the special case of each of the generating functions $\vartheta_{\lambda}(z, t ; s, a)$ and $\varphi(z, t ; s, a)$ in (1.11) and (1.12) when $z=a=1$ was investigated by Katsurada (1997). Subsequently, various results involving the generating functions $\vartheta_{\lambda}(z, t ; s, a)$ and $\varphi(z, t ; s, a)$ defined by (1.11) and (1.12), respectively, together with their such partial sums as those given by (1.13) to (1.16), were derived by Bin-Saad (2007) (see also the more recent sequels to (Bin-Saad 2007) and (Katsurada 1997) by Gupta and Kumari (2011) and by Saxena et al. (2011a).

Our main objective in this paper is to investigate, in a rather systematic manner, much more general families of generating functions and their partial sums than those associated with the generating functions $\vartheta_{\lambda}(z, t ; s, a)$ and $\varphi(z, t ; s, a)$ defined by (1.11) and (1.12), respectively. We also show the hitherto unnoticed fact that the so-called $\tau$-generalized Riemann Zeta function, which happens to be the main subject of investigation by Gupta and Kumari (2011) and by Saxena et al. (2011a), is simply a seemingly trivial notational variation of the familiar general
Hurwitz-Lerch Zeta function $\Phi(z, s, a)$ defined by (1.1). Finally, we present a sum-integral representation formula for the general family of the extended Hurwitz-Lerch Zeta functions.

\section{Families of the extended Hurwitz-Lerch Zeta functions and related special functions}

We begin this section by recalling the following sumintegral representation given by Yen et al. ((2002), p. 100, Theorem) for the Hurwitz (or generalized) Zeta function $\zeta(s, a)$ defined by (1.3):

$$
\zeta(s, a)=\frac{1}{\Gamma(s)} \sum_{j=0}^{k-1} \int_{0}^{\infty} \frac{t^{s-1} \mathrm{e}^{-(a+j) t}}{1-\mathrm{e}^{-k t}} \mathrm{~d} t
$$

$$
(k \in \mathbb{N} ; \Re(s)>1 ; \Re(a)>0),
$$

which, for $k=2$, was derived earlier by Nishimoto et al. ((2002), p. 94, Theorem 4). The following straightforward generalization of the sum-integral representation (2.1) involving the familiar general Hurwitz-Lerch Zeta function $\Phi(z, s, a)$ defined by (1.1) was given by Lin and Srivastava (2004, p. 727, Equation (7)):

$$
\begin{aligned}
& \Phi(z, s, a)=\frac{1}{\Gamma(s)} \sum_{j=0}^{k-1} z^{j} \int_{0}^{\infty} \frac{t^{s-1} \mathrm{e}^{-(a+j) t}}{1-z^{k} \mathrm{e}^{-k t}} \mathrm{~d} t \\
& (k \in \mathbb{N} ; \Re(a)>0 ; \Re(s)>0 \quad \text { when } \\
& |z| \leqq 1(z \neq 1) ; \Re(s)>1 \quad \text { when } \quad z=1) .
\end{aligned}
$$

The sum-integral representations (2.1) and (1.2) led Lin and Srivastava (2004) to the introduction and investigation of an interesting generalization of the Hurwitz-Lerch Zeta function $\Phi(z, s, a)$ in the following form given by Lin and Srivastava ((2004), p. 727, Equation (8)):

$$
\Phi_{\mu, v}^{(\rho, \sigma)}(z, s, a):=\sum_{n=0}^{\infty} \frac{(\mu)_{\rho n}}{(v)_{\sigma n}} \frac{z^{n}}{(n+a)^{s}}
$$

$\left(\mu \in \mathbb{C} ; a, v \in \mathbb{C} \backslash \mathbb{Z}_{0}^{-} ; \rho, \sigma \in \mathbb{R}^{+} ; \rho<\sigma \quad\right.$ when $s, z \in \mathbb{C} ; \rho=\sigma$ and $s \in \mathbb{C}$ when $|z|<\delta:=\rho^{-\rho} \sigma^{\sigma}$; $\rho=\sigma \quad$ and $\Re(s-\mu+\nu)>1$ when $|z|=\delta)$,

where $(\lambda)_{\nu}$ denotes the Pochhammer symbol defined, in terms of the familiar Gamma function, by (1.8). Clearly, we find from the definition (2.3) that

$$
\Phi_{\nu, v}^{(\sigma, \sigma)}(z, s, a)=\Phi_{\mu, v}^{(0,0)}(z, s, a)=\Phi(z, s, a)
$$

and

$$
\begin{aligned}
& \Phi_{\mu, 1}^{(1,1)}(z, s, a)=\Phi_{\mu}^{*}(z, s, a):=\sum_{n=0}^{\infty} \frac{(\mu)_{n}}{n !} \frac{z^{n}}{(n+a)^{s}} \\
& \left(\mu \in \mathbb{C} ; a \in \mathbb{C} \backslash \mathbb{Z}_{0}^{-} ; s \in \mathbb{C} \text { when }|z|<1 ;\right. \\
& \Re(s-\mu)>1 \quad \text { when } \quad|z|=1),
\end{aligned}
$$


where, as already pointed out by Lin and Srivastava (2004), $\Phi_{\mu}^{*}(z, s, a)$ is a generalization of the Hurwitz-Lerch Zeta function considered by Goyal and Laddha ((1997), p. 100, Equation (1.5)). For further results involving these classes of generalized Hurwitz-Lerch Zeta functions, see the recent works by Garg et al. (2006) and Lin et al. (2006).

A generalization of the above-defined Hurwitz-Lerch Zeta functions $\Phi(z, s, a)$ and $\Phi_{\mu}^{*}(z, s, a)$ was studied, in the following form, by Garg et al. ((2008), p. 313, Equation (1.7)):

$$
\begin{aligned}
& \Phi_{\lambda, \mu ; \nu}(z, s, a):=\sum_{n=0}^{\infty} \frac{(\lambda)_{n}(\mu)_{n}}{(\nu)_{n} \cdot n !} \frac{z^{n}}{(n+a)^{s}} \\
& \left(\lambda, \mu \in \mathbb{C} ; \nu, a \in \mathbb{C} \backslash \mathbb{Z}_{0}^{-} ; s \in \mathbb{C} \text { when }|z|<1 ;\right. \\
& \Re(s+v-\lambda-\mu)>1 \text { when }|z|=1) .
\end{aligned}
$$

Various integral representations and two-sided bounding inequalities for $\Phi_{\lambda, \mu ; v}(z, s, a)$ can be found in the works by Garg et al. (2008) and [Jankov et al. (2011)], respectively. These latter authors [Jankov et al. (2011)] also considered the function $\Phi_{\lambda, \mu ; v}(z, s, a)$ as a special kind of Mathieu type $(\boldsymbol{a}, \boldsymbol{\lambda})$-series.

If we compare the definitions (2.3) and (2.6), we can easily observe that the function $\Phi_{\lambda, \mu ; \nu}(z, s, a)$ studied by Garg et al. (2008) does not provide a generalization of the function $\Phi_{\mu, v}^{(\rho, \sigma)}(z, s, a)$ which was introduced earlier by Lin and Srivastava (2004). Indeed, for $\lambda=1$, the function $\Phi_{\lambda, \mu ; \nu}(z, s, a)$ coincides with a special case of the function $\Phi_{\mu, v}^{(\rho, \sigma)}(z, s, a)$ when $\rho=\sigma=1$, that is,

$$
\Phi_{1, \mu ; \nu}(z, s, a)=\Phi_{\mu, v}^{(1,1)}(z, s, a) .
$$

Next, for the Riemann-Liouville fractional derivative operator $\mathcal{D}_{z}^{\mu}$ defined by (see, for example, Erdélyi et al. ((1954), p. 181), Samko et al. (1993) and (Kilbas et al. 2006, p. 70 et seq.))

$$
\begin{aligned}
& \mathcal{D}_{z}^{\mu}\{f(z)\} \\
& := \begin{cases}\frac{1}{\Gamma(-\mu)} \int_{0}^{z}(z-t)^{-\mu-1} f(t) d t \quad(\Re(\mu)<0) \\
\frac{d^{m}}{d z^{m}}\left\{\mathcal{D}_{z}^{\mu-m}\{f(z)\}\right\} & (m-1 \leqq \Re(\mu)<m(m \in \mathbb{N})),\end{cases}
\end{aligned}
$$

the following formula is well-known:

$$
\mathcal{D}_{z}^{\mu}\left\{z^{\lambda}\right\}=\frac{\Gamma(\lambda+1)}{\Gamma(\lambda-\mu+1)} z^{\lambda-\mu} \quad(\Re(\lambda)>-1),
$$

which, by virtue of the definitions (1.1) and (2.3), yields the following fractional derivative formula for the generalized
Hurwitz-Lerch Zeta function $\Phi_{\mu, v}^{(\rho, \sigma)}(z, s, a)$ with $\rho=\sigma$ [Lin and Srivastava ((2004), p. 730, Equation (24))]:

$$
\begin{gathered}
\mathcal{D}_{z}^{\mu-v}\left\{z^{\mu-1} \Phi\left(z^{\sigma}, s, a\right)\right\}=\frac{\Gamma(\mu)}{\Gamma(\nu)} z^{\nu-1} \Phi_{\mu, \nu}^{(\sigma, \sigma)}\left(z^{\sigma}, s, a\right) \\
\left(\Re(\mu)>0 ; \sigma \in \mathbb{R}^{+}\right) .
\end{gathered}
$$

In its particular case when $v=\sigma=1$, the fractional derivative formula (2.9) would reduce at once to the following form:

$$
\Phi_{\mu}^{*}(z, s, a)=\frac{1}{\Gamma(\mu)} \mathcal{D}_{z}^{\mu-1}\left\{z^{\mu-1} \Phi(z, s, a)\right\} \quad(\Re(\mu)>0),
$$

which (as already remarked by Lin and Srivastava (2004), p. 730) exhibits the interesting (and useful) fact that $\Phi_{\mu}^{*}(z, s, a)$ is essentially a Riemann-Liouville fractional derivative of the classical Hurwitz-Lerch function $\Phi(z, s, a)$. Moreover, it is easily deduced from the fractional derivative formula (2.8) that

$$
\begin{aligned}
\Phi_{\lambda, \mu ; v}(z, s, a)= & \frac{\Gamma(v)}{\Gamma(\lambda)} z^{1-\lambda} \mathcal{D}_{z}^{\lambda-v}\left\{z^{\lambda-1} \Phi_{\mu}^{*}(z, s, a)\right\} \\
= & \frac{\Gamma(v)}{\Gamma(\lambda) \Gamma(\mu)} z^{1-\lambda} \mathcal{D}_{z}^{\lambda-v} \\
& \cdot\left\{z^{\lambda-1} \mathcal{D}_{z}^{\mu-1}\left\{z^{\mu-1} \Phi_{\mu}(z, s, a)\right\}\right\}
\end{aligned}
$$

which (as observed recently by Srivastava et al. (2011), pp. 490-491) exhibits the fact that the function $\Phi_{\lambda, \mu ; v}(z, s, a)$ studied by Garg et al. (2008) is essentially a consequence of the classical Hurwitz-Lerch Zeta function $\Phi(z, s, a)$ when we apply the Riemann-Liouville fractional derivative operator $\mathcal{D}_{z}^{\mu}$ two times as indicated above in (2.11). The interested reader may be referred also to many other explicit representations for $\Phi_{\mu}^{*}(z, s, a)$ and $\Phi_{\mu, v}^{(\rho, \sigma)}(z, s, a)$, which were proven by Lin and Srivastava (2004), including (for example) a potentially useful Eulerian integral representation of the first kind [Lin and Srivastava ((2004), p. 731, Equation (28))].

It should be remarked here that a multiple (or, simply, $n$ dimentional) Hurwitz-Lerch Zeta function $\Phi_{n}(z, s, a)$ was studied recently by Choi et al. ((2008), p. 66, Eq. (6)). On the other hand, Răducanu and Srivastava (see (Răducanu and Srivastava 2007), the references cited therein as well as many sequels thereto) made use of the HurwitzLerch Zeta function $\Phi(z, s, a)$ in defining a certain linear convolution operator in their systematic investigation of various analytic function classes in Geometric Function Theory in Complex Analysis. Furthermore, Gupta 
et al. (2008) revisited the study of the familiar HurwitzLerch Zeta distribution by investigating its structural properties, reliability properties and statistical inference. These investigations by Gupta et al. (2008) and others (see, for example, (Srivastava 2000), Srivastava and Choi (2001) and Srivastava et al. (2010); see also Saxena et al. (2011b) and Srivastava et al. (2011)), fruitfully using the Hurwitz-Lerch Zeta function $\Phi(z, s, a)$ and some of its above-mentioned generalizations, have led eventually to the following definition a family of the extended (multiparameter) Hurwitz-Lerch Zeta functions by Srivastava et al. (2011).

Definition 1. (Srivastava et al. (2011)). The family of the extended (multi-parameter) Hurwitz-Lerch Zeta functions

$$
\Phi_{\lambda_{1}, \cdots, \lambda_{p} ; \mu_{1}, \cdots, \mu_{q}}^{\left(\rho_{1}, \cdots, \rho_{p}, \sigma_{1}, \cdots, \sigma_{q}\right)}(z, s, a)
$$

is defined by

$$
\begin{aligned}
\Phi_{\lambda_{1}, \cdots, \lambda_{p}, \mu_{1}, \cdots, \mu_{q}}^{\left(\rho_{1}, \cdots, \rho_{p}, \sigma_{1}, \cdots, \sigma_{q}\right)}(z, s, a) & :=\sum_{n=0}^{\infty} \frac{\prod_{j=1}^{p}\left(\lambda_{j}\right)_{n \rho_{j}}}{n ! \prod_{j=1}^{q}\left(\mu_{j}\right)_{n \sigma_{j}}} \frac{z^{n}}{(n+a)^{s}} \\
= & : \sum_{n=0}^{\infty} \Xi_{n} \frac{z^{n}}{(n+a)^{s}}
\end{aligned}
$$

$\left(p, q \in \mathbb{N}_{0} ; \lambda_{j} \in \mathbb{C}(j=1, \cdots, p) ; a, \mu_{j} \in \mathbb{C} \backslash Z_{0}^{-}(j=1, \cdots, q) ;\right.$ $\rho_{j}, \sigma_{k} \in \mathbb{R}^{+}(j=1, \cdots, p ; k=1, \cdots, q) ; \Delta>-1 \quad$ when $s, z \in \mathbb{C} ; \Delta=-1$ and $s \in \mathbb{C}$ when $|z|<\nabla^{*} ; \Delta=-1$ and $\Re(\Xi)>\frac{1}{2} \quad$ when $\left.|z|=\nabla^{*}\right)$

where the sequence $\left\{\Xi_{n}\right\}_{n \in \mathbb{N}_{0}}$ of the coefficients in the definition (2.12) is given, for latter convenience, by

$$
\Xi_{n}:=\frac{\prod_{j=1}^{p}\left(\lambda_{j}\right)_{n \rho_{j}}}{n ! \prod_{j=1}^{q}\left(\mu_{j}\right)_{n \sigma_{j}}} \quad\left(n \in \mathbb{N}_{0}\right),
$$

$(\lambda)_{v} \quad(\lambda, v \in \mathbb{C})$ denotes the Pochhammer symbol given by (1.8) and

$$
\begin{aligned}
& \Delta:=\sum_{j=1}^{q} \sigma_{j}-\sum_{j=1}^{p} \rho_{j}, \\
& \Xi:=s+\sum_{j=1}^{q} \mu_{j}-\sum_{j=1}^{p} \lambda_{j}+\frac{p-q}{2}
\end{aligned}
$$

and

$$
\nabla^{*}:=\left(\prod_{j=1}^{p} \rho_{j}^{-\rho_{j}}\right) \cdot\left(\prod_{j=1}^{q} \sigma_{j}^{\sigma_{j}}\right) .
$$

In order to derive direct relationships of the family of the extended (multi-parameter) Hurwitz-Lerch Zeta functions

$$
\Phi_{\lambda_{1}, \cdots, \lambda_{p} ; \mu_{1}, \cdots, \mu_{q}}^{\left(\rho_{1}, \cdots, \rho_{p}, \sigma_{1}, \cdots, \sigma_{q}\right)}(z, s, a)
$$

defined by (2.12) with several other relatively more familiar special functions, we need each of the following definitions.

Definition 2. The Fox-Wright function $\Psi_{q} \quad\left(p, q \in \mathbb{N}_{0}\right)$ or $p \Psi_{q}^{*} \quad\left(p, q \in \mathbb{N}_{0}\right)$, which is a further generalization of the familiar generalized hypergeometric function ${ }_{p} F_{q} \quad\left(p, q \in \mathbb{N}_{0}\right)$, with $p$ numerator parameters $a_{1}, \cdots, a_{p}$ and $q$ denominator parameters $b_{1}, \cdots, b_{q}$ such that

$$
a_{j} \in \mathbb{C}(j=1, \cdots, p)
$$

and

$$
b_{j} \in \mathbb{C} \backslash \mathbb{Z}_{0}^{-}(j=1, \cdots, q),
$$

defined by (see, for details, (Erdélyi et al. 1953, p. 183) and (Choi et al. 1985, p. 21); see also (Kilbas et al. 2006, p. 56), (Choi et al. 2010, p. 30) and (Srivastava et al. 1982, p. 19))

$$
\begin{aligned}
{ }_{p} \Psi_{q}^{*}\left[\begin{array}{c}
\left(a_{1}, A_{1}\right), \cdots,\left(a_{p}, A_{p}\right) ; \\
\left(b_{1}, B_{1}\right), \cdots,\left(b_{q}, B_{q}\right) ;
\end{array}\right] \\
\quad:=\sum_{n=0}^{\infty} \frac{\left(a_{1}\right)_{A_{1} n} \cdots\left(a_{p}\right)_{A_{p} n}}{\left(b_{1}\right)_{B_{1} n} \cdots\left(b_{q}\right)_{B_{q} n}} \frac{z^{n}}{n !} \\
\quad=\frac{\Gamma\left(b_{1}\right) \cdots \Gamma\left(b_{q}\right)}{\Gamma\left(a_{1}\right) \cdots \Gamma\left(a_{p}\right)}{ }_{p} \Psi_{q}\left[\begin{array}{c}
\left(a_{1}, A_{1}\right), \cdots,\left(a_{p}, A_{p}\right) ; \\
\left(b_{1}, B_{1}\right), \cdots,\left(b_{q}, B_{q}\right) ;
\end{array}\right]
\end{aligned}
$$

$$
\begin{aligned}
& \left(A_{j}>0 \quad(j=1, \cdots, p) ; B_{j}>0\right. \\
& \left.(j=1, \cdots, q) ; 1+\sum_{j=1}^{q} B_{j}-\sum_{j=1}^{p} A_{j} \geqq 0\right),
\end{aligned}
$$

where the equality in the convergence condition holds true for suitably bounded values of $|z|$ given by

$$
|z|<\nabla:=\left(\prod_{j=1}^{p} A_{j}^{-A_{j}}\right) \cdot\left(\prod_{j=1}^{q} B_{j}^{B_{j}}\right) .
$$

In the particular case when

$$
A_{j}=B_{k}=1 \quad(j=1, \cdots, p ; k=1, \cdots, q),
$$


we have the following relationship (see, for details, (Choi et al. 1985, p. 21)):

$$
\begin{aligned}
p & \Psi_{q}^{*}\left[\begin{array}{c}
\left(a_{1}, 1\right), \cdots,\left(a_{p}, 1\right) ; \\
\left(b_{1}, 1\right), \cdots,\left(b_{q}, 1\right) ;
\end{array}\right] \\
& ={ }_{p} F_{q}\left[\begin{array}{c}
a_{1}, \cdots, a_{p} ; \\
z \\
b_{1}, \cdots, b_{q} ;
\end{array}\right] \\
& =\frac{\Gamma\left(b_{1}\right) \cdots \Gamma\left(b_{q}\right)}{\Gamma\left(a_{1}\right) \cdots \Gamma\left(a_{p}\right)} p_{p} \Psi_{q}\left[\begin{array}{c}
\left(a_{1}, 1\right), \cdots,\left(a_{p}, 1\right) ; \\
\left(b_{1}, 1\right), \cdots,\left(b_{q}, 1\right) ;
\end{array}\right],
\end{aligned}
$$

in terms of the generalized hypergeometric function ${ }_{p} F_{q} \quad\left(p, q \in \mathbb{N}_{0}\right)$.

Definition 3. An attempt to derive Feynman integrals in two different ways, which arise in perturbation calculations of the equilibrium properties of a magnetic mode of phase transitions, led naturally to the following generalization of Fox's $H$-function (Inayat-Hussain 1987b, p. 4126) (see also (Buschman and Srivastava 1990) and (Inayat-Hussain 1987a)):

$$
\begin{aligned}
& \bar{H}(z)=\bar{H}_{p, q}^{m, n}[z] \\
& =\bar{H}_{p, q}^{m, n}\left[z \mid \begin{array}{l}
\left(a_{j}, A_{j} ; \alpha_{j}\right)_{j=1}^{n},\left(a_{j}, A_{j}\right)_{j=n+1}^{p} \\
\left.b_{j}, B_{j}\right)_{j=1}^{m},\left(b_{j}, B_{j} ; \beta_{j}\right)_{j=m+1}^{q}
\end{array}\right] \\
& :=\frac{1}{2 \pi \mathrm{i}} \int_{\mathfrak{L}} \chi(s) z^{s} \mathrm{~d} s \\
& (z \neq 0 ; \mathrm{i}=\sqrt{-1} ; \chi(s) \\
& \left.:=\frac{\prod_{j=1}^{m} \Gamma\left(b_{j}-B_{j} s\right) \cdot \prod_{j=1}^{n}\left\{\Gamma\left(1-a_{j}+A_{j} s\right)\right\}^{\alpha_{j}}}{\prod_{j=n+1}^{p} \Gamma\left(a_{j}-A_{j} s\right) \cdot \prod_{j=m+1}^{q}\left\{\Gamma\left(1-b_{j}+B_{j} s\right)\right\}^{\beta_{j}}}\right),
\end{aligned}
$$

which contains fractional powers of some of the Gamma functions involved. Here, and in what follows, the parameters

$$
A_{j}>0(j=1, \cdots, p) \text { and } B_{j}>0(j=1, \cdots, q),
$$

the exponents

$$
\alpha_{j} \quad(j=1, \cdots, n) \quad \text { and } \quad \beta_{j} \quad(j=m+1, \cdots, q)
$$

can take on noninteger values, and $\mathfrak{L}=\mathfrak{L}_{(\mathrm{i} \tau ; \infty)}$ is a MellinBarnes type contour starting at the point $\tau-\mathrm{i} \infty$ and terminating at the point $\tau+\mathrm{i} \infty(\tau \in \mathbb{R})$ with the usual indentations to separate one set of poles from the other set of poles. The sufficient condition for the absolute convergence of the contour integral in (2.18) was established as follows by Buschman and Srivastava ((1990), p. 4708):

$$
\Lambda:=\sum_{j=1}^{m} B_{j}+\sum_{j=1}^{n}\left|\alpha_{j}\right| A_{j}-\sum_{j=m+1}^{q}\left|\beta_{j}\right| B_{j}-\sum_{j=n+1}^{p} A_{j}>0,
$$

which provides exponential decay of the integrand in (2.18) and the region of absolute convergence of the contour integral in (2.18) is given by

$$
|\arg (z)|<\frac{1}{2} \pi \Lambda,
$$

where $\Lambda$ is defined by (2.19).

Remark 1. If we set

$$
s=0, \quad p \mapsto p+1 \quad\left(\rho_{1}=\cdots=\rho_{p}=1 ; \quad \lambda_{p+1}=\rho_{p+1}=1\right)
$$

and

$$
q \mapsto q+1 \quad\left(\sigma_{1}=\cdots=\sigma_{q}=1 ; \quad \mu_{q+1}=\beta ; \quad \sigma_{q+1}=\alpha\right),
$$

then (2.12) reduces to the following generalized $M$-series which was recently introduced by Sharma and Jain (2009) (see also an earlier paper by Sharma (2008) for the special case when $\beta=1$ ):

$$
\begin{aligned}
& { }_{p}^{\alpha, \beta} M_{q}\left(a_{1}, \cdots, a_{p} ; b_{1}, \cdots, b_{q} ; z\right) \\
& \quad:=\sum_{k=0}^{\infty} \frac{\left(a_{1}\right)_{k} \cdots\left(a_{p}\right)_{k}}{\left(b_{1}\right)_{k} \cdots\left(b_{q}\right)_{k}} \frac{z^{k}}{\Gamma(\alpha k+\beta)} \\
& \quad=\frac{1}{\Gamma(\beta)}{ }_{p+1} \Psi_{q+1}^{*}\left[\begin{array}{c}
\left(a_{1}, 1\right), \cdots,\left(a_{p}, 1\right),(1,1) ; \\
\left(b_{1}, 1\right), \cdots,\left(b_{q}, 1\right),(\beta, \alpha) ;
\end{array}\right],
\end{aligned}
$$

in which the last relationship exhibits the fact that the so-called generalized $M$-series is indeed an obvious special case of the Fox-Wright function $p \Psi_{q}^{*}$ defined by (2.15) (see also (Saxana 2009)). Similarly, for the generalized Mittag-Leffler function considered by Kilbas et al. (2002), we have

$$
\begin{aligned}
E_{\rho}\left[\left(\beta_{1}, \eta_{1}\right), \cdots,\left(\beta_{q}, \eta_{q}\right) ; z\right] & \\
& :=\sum_{k=0}^{\infty} \frac{(\rho)_{k}}{\prod_{j=1}^{q} \Gamma\left(\eta_{j} k+\beta_{j}\right)} \\
& =\frac{1}{\Gamma(\rho)}{ }_{1} \Psi_{q}\left[\begin{array}{r}
(\rho, 1) ; \\
z \\
\left(\beta_{1}, \eta_{1}\right), \cdots,\left(\beta_{q}, \eta_{q}\right) ;
\end{array}\right],
\end{aligned}
$$


Remark 2. The following $\bar{H}$-function representation can be applied in order to derive various properties of the extended Hurwitz-Lerch Zeta function

$$
\Phi_{\lambda_{1}, \cdots, \lambda_{p} ; \mu_{1}, \cdots, \mu_{q}}^{\left(\rho_{1}, \cdots, \rho_{p}, \sigma_{1}, \cdots, \sigma_{q}\right)}(z, s, a)
$$

from those of the $\bar{H}$-function (see, for details, Srivastava et al. ((2011), p. 504, Theorem 8)):

$$
\begin{aligned}
& \Phi_{\lambda_{1}, \cdots, \lambda_{p} ; \mu_{1}, \cdots, \mu_{q}}^{\left(\rho_{1}, \cdots, \rho_{p}, \sigma_{1}, \cdots, \sigma_{q}\right)}(z, s, a) \\
= & \frac{\prod_{j=1}^{q} \Gamma\left(\mu_{j}\right)}{\prod_{j=1}^{p} \Gamma\left(\lambda_{j}\right)} \bar{H}_{p+1, q+2}^{1, p+1} \\
& {\left[\begin{array}{l}
\prod_{j}(0,1),\left(1-\mu_{1}, \sigma_{1} ; 1\right), \cdots,\left(1-\mu_{q}, \sigma_{q} ; 1\right),(-a, 1 ; s)
\end{array}\right] } \\
= & \frac{\prod_{j=1}^{q} \Gamma\left(\mu_{j}\right)}{\prod_{j=1}^{p} \Gamma\left(\lambda_{j}\right)} \cdot \frac{1}{2 \pi \mathrm{i}} \int_{\mathcal{L}} \frac{\Gamma(-\xi)\{\Gamma(\xi+a)\}^{s} \prod_{j=1}^{p} \Gamma\left(\lambda_{j}+\rho_{j} \xi\right)}{\{\Gamma(\xi+a+1)\}^{s} \prod_{j=1}^{q} \Gamma\left(\mu_{j}+\sigma_{j} \xi\right)}(-z)^{\xi} \mathrm{d} \xi \\
& (|\arg (-z)|<\pi),
\end{aligned}
$$

the path of integration $\mathcal{L}$ in the last member of (2.23) being a Mellin-Barnes type contour in the complex $\xi$ plane, which starts at the point $-\mathrm{i} \infty$ and terminates at the point $\mathrm{i} \infty$ with indentations, if necessary, in such a manner as to separate the poles of $\Gamma(-\xi)$ from the poles of $\Gamma\left(\lambda_{j}+\rho_{j} \xi\right) \quad(j=1, \cdots, p)$. Thus, for example, by making use of a known fractional-calculus result due to Srivastava et al. ((2006), p. 97, Equation (2.4)), we readily obtain the following extension of such fractional derivative formulas as (2.9) and (2.10) [Srivastava et al. ((2011), p. 505, Equation (6.8))]:

\section{Generating relations associated with the extended Hurwitz-Lerch Zeta function}

In this section, we first introduce the following generating functions and their partial sums involving the extended Hurwitz-Lerch Zeta function

$$
\Phi_{\lambda_{1}, \cdots, \lambda_{p} ; \mu_{1}, \cdots, \mu_{q}}^{\left(\rho_{1}, \cdots, \rho_{p}, \sigma_{1}, \cdots, \sigma_{q}\right)}(z, s, a)
$$

defined by (2.12). Indeed, as a generalization of the generating functions (1.9) and (1.10), we have

$$
\begin{aligned}
& \Phi_{\lambda_{1}, \cdots, \lambda_{p} ; \mu_{1}, \cdots, \mu_{q}}^{\left(\rho_{1}, \cdots, \rho_{q}, \sigma_{1}, \cdots, \sigma_{q}\right)}(z, s, a-t) \\
& \quad=\sum_{n=0}^{\infty} \frac{(s)_{n}}{n !} \Phi_{\lambda_{1}, \cdots, \lambda_{p} ; \mu_{1}, \cdots, \mu_{q}}^{\left(\rho_{1}, \cdots, \rho_{p}, \sigma_{1}, \cdots, \sigma_{q}\right)}(z, s+n, a) t^{n} \quad(|t|<|a|),
\end{aligned}
$$

which can easily be put in the following more general form:

$$
\begin{aligned}
& \sum_{n=0}^{\infty} \frac{(\lambda)_{n}}{n !} \Phi_{\lambda_{1}, \cdots, \lambda_{p} ; \mu_{1}, \cdots, \mu_{q}}^{\left(\rho_{1}, \cdots, \rho_{p}, \sigma_{1}, \cdots, \sigma_{q}\right)}(z, s+n, a) t^{n} \\
& \quad=\sum_{k=0}^{\infty} \frac{\Xi_{k} z^{k}}{(k+a)^{s-\lambda}(k+a-t)^{\lambda}}=: \Omega_{\lambda}(z, t ; s, a) \quad(|t|<|a|),
\end{aligned}
$$

where the sequence $\left\{\Xi_{n}\right\}_{n \in \mathbb{N}_{0}}$ of the coefficients in (2.12) is given by (2.13). This last generating function (3.2) would reduce immediately to the expansion formula (4.1) in its special case when $\lambda=s$. Furthermore, in its limit case when

$$
t \mapsto \frac{t}{\lambda} \quad \text { and } \quad|\lambda| \rightarrow \infty,
$$

the generating function (3.2) yields

$$
\begin{aligned}
& \sum_{n=0}^{\infty} \Phi_{\lambda_{1}, \cdots, \lambda_{p} ; \mu_{1}, \cdots, \mu_{q}}^{\left(\rho_{1}, \cdots, \rho_{p}, \sigma_{1}, \cdots, \sigma_{q}\right)}(z, s+n, a) \frac{t^{n}}{n !} \\
& \quad=\sum_{k=0}^{\infty} \frac{\Xi_{k} z^{k}}{(k+a)^{s}} \exp \left(\frac{t}{k+a}\right)=: \Theta(z, t ; s, a) \quad(|t|<\infty),
\end{aligned}
$$

$$
\begin{aligned}
& \mathcal{D}_{z}^{\nu-\tau}\left\{z^{\nu-1} \Phi_{\lambda_{1}, \cdots, \lambda_{p} ; \mu_{1}, \cdots, \mu_{q}}^{\left(\rho_{1}, \cdots, \rho_{p}, \sigma_{1}, \cdots, \sigma_{q}\right)}\left(z^{\kappa}, s, a\right)\right\}=\frac{\prod_{j=1}^{q} \Gamma\left(\mu_{j}\right)}{\prod_{j=1}^{p} \Gamma\left(\lambda_{j}\right)} z^{\tau-1} \\
& \cdot \bar{H}_{p+2, q+3}^{1, p+2}\left[-z^{\kappa} \mid \begin{array}{c}
\left(1-\lambda_{1}, \rho_{1} ; 1\right), \cdots,\left(1-\lambda_{p}, \rho_{p} ; 1\right),(1-v, \kappa ; 1),(1-a, 1 ; s) \\
(0,1),\left(1-\mu_{1}, \sigma_{1} ; 1\right), \cdots,\left(1-\mu_{q}, \sigma_{q} ; 1\right),(1-\tau, \kappa ; 1),(-a, 1 ; s)
\end{array}\right] \\
& =\frac{\Gamma(v)}{\Gamma(\tau)} z^{\tau-1} \Phi_{\lambda_{1}, \cdots, \lambda_{p}, \nu ; \mu_{1}, \cdots, \mu_{q}, \tau}^{\left(\rho_{1}, \cdots, \rho_{p}, \kappa, \sigma_{1}, \cdots, \sigma_{q}, \kappa\right)}\left(z^{\kappa}, s, a\right) \quad(\Re(\nu)>0 ; \kappa>0) \text {. }
\end{aligned}
$$


where the sequence $\left\{\Xi_{n}\right\}_{n \in \mathbb{N}_{0}}$ of the coefficients in (2.12) is given, as before, by (2.13).

We shall also consider each of the following truncated forms of the generating functions $\Omega_{\lambda}(z, t ; s, a)$ and $\Theta(z, t ; s, a)$ in (3.2) and (3.3), respectively:

$$
\begin{aligned}
& \Omega_{\lambda}^{(0, r)}(z, t ; s, a):=\sum_{k=0}^{r} \frac{\Xi_{k} z^{k}}{(k+a)^{s-\lambda}(k+a-t)^{\lambda}} \quad\left(r \in \mathbb{N}_{0}\right), \\
& \Omega_{\lambda}^{(r+1, \infty)}(z, t ; s, a):=\sum_{k=r+1}^{\infty} \frac{\Xi_{k} z^{k}}{(k+a)^{s-\lambda}(k+a-t)^{\lambda}} \quad\left(r \in \mathbb{N}_{0}\right), \\
& \Theta^{(0, r)}(z, t ; s, a):=\sum_{k=0}^{r} \frac{\Xi_{k} z^{k}}{(k+a)^{s}} \exp \left(\frac{t}{k+a}\right) \quad\left(r \in \mathbb{N}_{0}\right)
\end{aligned}
$$

and

$$
\Theta^{(r+1, \infty)}(z, t ; s, a):=\sum_{k=r+1}^{\infty} \frac{\Xi_{k} z^{k}}{(k+a)^{s}} \exp \left(\frac{t}{k+a}\right) \quad\left(r \in \mathbb{N}_{0}\right),
$$

which obviously satisfy the following decomposition formulas:

$$
\Omega_{\lambda}^{(0, r)}(z, t ; s, a)+\Omega_{\lambda}^{(r+1, \infty)}(z, t ; s, a)=\Omega_{\lambda}(z, t ; s, a)
$$

and

$$
\Theta^{(0, r)}(z, t ; s, a)+\Theta^{(r+1, \infty)}(z, t ; s, a)=\Theta(z, t ; s, a) .
$$

Our first set of integral representations for the abovedefined generating functions is contained in Theorem 1 below.

Theorem 1. Each of the following integral representation formulas holds true:

$$
\begin{aligned}
\Omega_{\lambda}(z, \omega ; s, a)= & \frac{1}{\Gamma(s)} \int_{0}^{\infty} t^{s-1} \mathrm{e}^{-a t}{ }_{p} \Psi_{q}^{*} \\
& {\left[\begin{array}{c}
\left(\lambda_{1}, \rho_{1}\right), \cdots,\left(\lambda_{p}, \rho_{p}\right) ; z \mathrm{e}^{-t} \\
\left(\mu_{1}, \sigma_{1}\right), \cdots,\left(\mu_{q}, \sigma_{q}\right) ;
\end{array}\right] } \\
& \cdot{ }_{1} F_{1}(\lambda ; s ; \omega t) \mathrm{d} t \quad(\min \{\Re(a), \Re(s)\}>0)
\end{aligned}
$$

and

$$
\begin{aligned}
\Theta(z, \omega ; s, a)= & \frac{1}{\Gamma(s)} \int_{0}^{\infty} t^{s-1} \mathrm{e}^{-a t}{ }_{p} \Psi_{q}^{*} \\
& {\left[\begin{array}{c}
\left(\lambda_{1}, \rho_{1}\right), \cdots,\left(\lambda_{p}, \rho_{p}\right) ; \\
\left(\mu_{1}, \sigma_{1}\right), \cdots,\left(\mu_{q}, \sigma_{q}\right) ;
\end{array}\right] } \\
& \cdot{ }_{0} F_{1}(-; s ; \omega t) \mathrm{d} t \quad(\min \{\Re(a), \Re(s)\}>0),
\end{aligned}
$$

provided that both sides of each of the assertions (3.10) and (3.11) exist.

Proof. For convenience, we denote by $\mathfrak{S}$ the second member of the assertion (3.10) of Theorem 1. Then, upon expanding the functions ${ }_{p} \Psi_{q}^{*}$ and ${ }_{1} F_{1}$ in series forms, we find that

$$
\begin{aligned}
\mathfrak{S}: & =\frac{1}{\Gamma(s)} \int_{0}^{\infty} t^{s-1} \mathrm{e}^{-a t}{ }_{p} \Psi_{q}^{*} \\
& {\left[\begin{array}{c}
\left(\lambda_{1}, \rho_{1}\right), \cdots,\left(\lambda_{p}, \rho_{p}\right) ; \\
\left(\mu_{1}, \sigma_{1}\right), \cdots,\left(\mu_{q}, \sigma_{q}\right) ;
\end{array} \mathrm{e}^{-t}\right]{ }_{1} F_{1}(\lambda ; s ; \omega t) \mathrm{d} t } \\
& =\frac{1}{\Gamma(s)} \sum_{m, n=0}^{\infty} \Xi_{m} z^{m} \frac{(\lambda)_{n}}{(s)_{n}} \frac{\omega^{n}}{n !} \int_{0}^{\infty} t^{s+n-1} \mathrm{e}^{-(a+m) t} \mathrm{~d} t,
\end{aligned}
$$

where the inversion of the order of integration and double summation can easily be justified by absolute convergence under the conditions stated with (3.10), $\Xi_{n}$ being defined by (2.13). Now, if we evaluate the innermost integral in (3.12) by appealing to the following well-known result:

$$
\int_{0}^{\infty} t^{\mu-1} \mathrm{e}^{-\kappa t} \mathrm{~d} t=\frac{\Gamma(\mu)}{\kappa^{\mu}} \quad(\min \{\Re(\kappa), \Re(\mu)\}>0),
$$

we get

$$
\begin{aligned}
\mathfrak{S}= & \sum_{n=0}^{\infty} \frac{(\lambda)_{n}}{n !}\left(\sum_{m=0}^{\infty} \Xi_{m} \frac{z^{m}}{(m+a)^{s+n}}\right) \omega^{n} \\
& (\min \{\Re(a), \Re(s)\}>0),
\end{aligned}
$$

which, in light of the definitions (2.12) and (3.2), yields the left-hand side of the first assertion (3.10) of Theorem 1.

The second assertion (3.11) of Theorem 1 can be proven in a similar manner.

Remark 3. For $\omega=0$, each of the assertions (3.10) and (3.11) of Theorem 1 yields a known integral representation formula due to Srivastava et al. ((2011), p. 504, Equation (6.4)). Moreover, in their special case when

$$
\omega=0 \quad \text { and } \quad \Xi_{n}=1 \quad\left(n \in \mathbb{N}_{0}\right),
$$

the assertions (3.10) and (3.11) of Theorem 1 would reduce immediately to the classical integral representation (1.7) for the Hurwitz-Lerch Zeta function $\Phi(z, s, a)$.

The proof of Theorem 2 below would run parallel to that of Theorem 1, which we already have detailed above fairly adequately. It is based essentially upon the Hankel type 
contour integral in the following form (Erdélyi et al. 1953, p. 14, Equation 1.16 (4)):

$$
\begin{aligned}
2 \mathrm{i} \sin (\pi v) \Gamma(v)= & -\int_{\infty}^{(0+)}(-t)^{\nu-1} \mathrm{e}^{-t} \mathrm{~d} t \\
(|\arg (-t)| \leqq \pi) &
\end{aligned}
$$

or, equivalently,

$$
\begin{gathered}
\frac{1}{\Gamma(1-v)}=-\frac{1}{2 \pi \mathrm{i}} \int_{\infty}^{(0+)}(-t)^{\nu-1} \mathrm{e}^{-t} \mathrm{~d} t \\
(|\arg (-t)| \leqq \pi) .
\end{gathered}
$$

Theorem 2. Each of the following Hankel type contour integral representation formulas holds true:

$$
\begin{aligned}
\Omega_{\lambda}(z, \omega ; s, a)= & -\frac{\Gamma(1-s)}{2 \pi \mathrm{i}} \int_{\infty}^{(0+)}(-t)^{s-1} \mathrm{e}^{-a t}{ }_{p} \Psi_{q}^{*} \\
& {\left[\begin{array}{l}
\left(\lambda_{1}, \rho_{1}\right), \cdots,\left(\lambda_{p}, \rho_{p}\right) ; \\
\left(\mu_{1}, \sigma_{1}\right), \cdots,\left(\mu_{q}, \sigma_{q}\right) ;
\end{array}\right] } \\
& \cdot \mathrm{e}^{-t} F_{1}(\lambda ; s ; \omega t) \mathrm{d} t \quad(\Re(a)>0 ;|\arg (-t)| \leqq \pi)
\end{aligned}
$$

and

$$
\begin{aligned}
\Theta(z, \omega ; s, a)= & -\frac{\Gamma(1-s)}{2 \pi \mathrm{i}} \int_{\infty}^{(0+)}(-t)^{s-1} \mathrm{e}^{-a t}{ }_{p} \Psi_{q}^{*} \\
& {\left[\begin{array}{c}
\left(\lambda_{1}, \rho_{1}\right), \cdots,\left(\lambda_{p}, \rho_{p}\right) ; \\
\left(\mu_{1}, \sigma_{1}\right), \cdots,\left(\mu_{q}, \sigma_{q}\right) ;
\end{array}\right] } \\
& \mathrm{e}^{-t} F_{1}(-; s ; \omega t) \mathrm{d} t(\Re(a)>0 ;|\arg (-t)| \leqq \pi),
\end{aligned}
$$

provided that both sides of each of the assertions (3.17) and (3.18) exist.

Remark 4. For $\omega=0$, each of the assertions (3.17) and (3.18) of Theorem 2 yields the following (presumably new) integral representation formula:

$$
\begin{aligned}
\Phi_{\lambda_{1}, \cdots, \lambda_{p} ; \mu_{1}, \cdots, \mu_{q}}^{\left(\rho_{1}, \cdots, \rho_{p}, \sigma_{1}\right)}(z, s, a) & \\
= & -\frac{\Gamma(1-s)}{2 \pi \mathrm{i}} \int_{\infty}^{(0+)}(-t)^{s-1} \mathrm{e}^{-a t} \\
& \cdot p \Psi_{q}^{*}\left[\begin{array}{c}
\left(\lambda_{1}, \rho_{1}\right), \cdots,\left(\lambda_{p}, \rho_{p}\right) ; \\
\left(\mu_{1}, \sigma_{1}\right), \cdots,\left(\mu_{q}, \sigma_{q}\right) ;
\end{array} \mathrm{e}^{-t}\right] \mathrm{d} t \\
& (\Re(a)>0 ;|\arg (-t)| \leqq \pi) .
\end{aligned}
$$

Furthermore, in their special case when

$$
\omega=0 \quad \text { and } \quad \Xi_{n}=1 \quad\left(n \in \mathbb{N}_{0}\right),
$$

the assertions (3.17) and (3.18) of Theorem 2 would reduce to the classical Hankel type contour integral representation for the Hurwitz-Lerch Zeta function $\Phi(z, s, a)$ (see, for example, (Erdélyi et al., 1953, p. 28, Equation 1.11 (5)); see also (Srivastava and Choi 2012), p. 195, Equation $2.5(8))$.

Next, by making use of the following known result (see, for example, Srivastava and Manocha ((1984), p. 86, Problem 1):

$$
\begin{array}{r}
\int_{a}^{b}(t-a)^{\alpha-1}(b-t)^{\beta-1} \mathrm{~d} t=(b-a)^{\alpha+\beta-1} B(\alpha, \beta) \\
(b \neq a ; \min \{\Re(\alpha), \Re(\beta)\}>0),
\end{array}
$$

we evaluate several Eulerian Beta-function integrals involving the generating functions $\Omega_{\lambda}(z, t ; s, a)$ and $\Theta(z, t ; s, a)$ defined by (3.2) and (3.3), respectively, $B(\alpha, \beta)$ being the familiar Beta function.

Theorem 3. In terms of the sequence $\left\{\Xi_{n}\right\}_{n \in \mathbb{N}_{0}}$ of the coefficients given by the definition (2.13), each of the following Eulerian Beta-function integral formulas holds true:

$$
\begin{gathered}
\int_{\xi}^{\eta}(t-\xi)^{\alpha-1}(\eta-t)^{\beta-1} \Omega_{\lambda}\left(z, \omega(t-\xi)^{\gamma}(\eta-t)^{\delta} ; s, a\right) \mathrm{d} t \\
=(\eta-\xi)^{\alpha+\beta-1} B(\alpha, \beta) \sum_{n=0}^{\infty} \Xi_{n} \frac{z^{n}}{(n+a)^{s}} 3_{3} \Psi_{1}^{*} \\
\cdot\left[\begin{array}{c}
(\lambda, 1),(\alpha, \gamma),(\beta, \delta) ; \\
\left.\quad \frac{\omega(\eta-\xi)^{\gamma+\delta}}{n+a}\right] \\
(\alpha+\beta, \gamma+\delta) ; \\
(\eta \neq \xi ; \min \{\Re(\alpha), \Re(\beta)\}>0 ; \gamma, \delta>0)
\end{array}\right.
\end{gathered}
$$

and

$$
\begin{gathered}
\int_{\xi}^{\eta}(t-\xi)^{\alpha-1}(\eta-t)^{\beta-1} \Theta\left(z, \omega(t-\xi)^{\gamma}(\eta-t)^{\delta} ; s, a\right) \mathrm{d} t \\
=(\eta-\xi)^{\alpha+\beta-1} B(\alpha, \beta) \sum_{n=0}^{\infty} \Xi_{n} \frac{z^{n}}{(n+a)^{s}} 2 \Psi_{1}^{*} \\
\cdot\left[\begin{array}{c}
(\alpha, \gamma),(\beta, \delta) ; \frac{\omega(\eta-\xi)^{\gamma+\delta}}{n+a} \\
(\alpha+\beta, \gamma+\delta) ; \\
(\eta \neq \xi ; \min \{\Re(\alpha), \Re(\beta)\}>0 ; \gamma, \delta>0),
\end{array}\right.
\end{gathered}
$$

provided that both sides of each of the assertions (3.21) and (3.22) exist, the Fox-Wright function $\mathrm{OO}_{3} \Psi_{1}^{*}$ in (3.21) being tacitly interpreted as an H-function contained in the definition (2.18).

Proof. Each of the assertions (3.21) and (3.22) of Theorem 3 can be proven fairly easily by appealing to the definitions (3.2) and (3.3), respectively, in conjunction 
with the Eulerian Beta-function integral (3.20). The details involved are being skipped here.

Remark 5. In addition to their relatively more familiar cases when $\xi=\eta-1=0$, various interesting limit cases of the integral formulas (3.21) and (3.22) asserted by Theorem 3 can be deduced by letting

$$
\lim _{\gamma \downarrow 0} \quad \text { or } \quad \lim _{\delta \downarrow 0} .
$$

Some such very specialized cases of Theorem 3 can be found in the recent works by Bin-Saad (2007), Gupta and Kumari (2011) and Saxena et al. (2011a).

The Eulerian Gamma-function integrals involving the generating functions $\Omega_{\lambda}(z, t ; s, a)$ and $\Theta(z, t ; s, a)$ defined by (3.2) and (3.3), respectively, which are asserted by Theorem 4 below, can be evaluated by applying the wellknown formula (3.13).

Theorem 4. Let the function $\Phi_{\mu}^{*}(z, s, a)$ be defined by (2.5). Then, in terms of the sequence $\left\{\Xi_{n}\right\}_{n \in \mathbb{N}_{0}}$ of the coefficients given by the definition (2.13), each of the following single or double Eulerian Gamma-function integral formulas holds true:

$$
\begin{aligned}
& \frac{1}{\Gamma(\mu)} \int_{0}^{\infty} t^{\mu-1} \mathrm{e}^{-\kappa t} \Omega_{\lambda}\left(z, \omega \mathrm{e}^{-\delta t} ; s, a\right) \mathrm{d} t \\
&= \delta^{-\mu} \sum_{n=0}^{\infty} \frac{\Xi_{n} z^{n}}{(n+a)^{s}} \Phi_{\lambda}^{*}\left(\frac{\omega}{n+a}, \mu, \frac{\kappa}{\delta}\right) \quad(3.23) \\
&(\min \{\Re(\kappa), \Re(\mu), \Re(\delta)\}>0), \\
& \frac{1}{\Gamma(\mu)} \int_{0}^{\infty} t^{\mu-1} \mathrm{e}^{-\kappa t} \Theta(z, \omega t ; s, a) \mathrm{d} t \\
&=\kappa^{-\mu} \Omega_{\mu}\left(z, \frac{\omega}{\kappa} ; s, a\right) \quad(\min \{\Re(\kappa), \Re(\mu)\}>0)
\end{aligned}
$$

and

$$
\begin{gathered}
\frac{1}{\Gamma(\mu) \Gamma(v)} \int_{0}^{\infty} \int_{0}^{\infty} u^{\mu-1} v^{v-1} \mathrm{e}^{-\kappa u-\delta v} \Theta\left(z, \omega u \mathrm{e}^{-\sigma v} ; s, a\right) \mathrm{d} u \mathrm{~d} v \\
=\kappa^{-\mu} \sigma^{-v} \sum_{n=0}^{\infty} \frac{\Xi_{n} z^{n}}{(n+a)^{s}} \Phi_{\mu}^{*}\left(\frac{\omega}{\kappa(n+a)}, \mu, \frac{\delta}{\sigma}\right) \\
(\min \{\Re(\kappa), \Re(\mu), \Re(v), \Re(\delta), \Re(\sigma)\}>0),
\end{gathered}
$$

provided that both sides of each of the assertions (3.23), (3.24) and (3.25) exist.

Remark 6. Some very specialized cases of Theorem 4 when

$$
\Xi_{n}=1 \quad\left(n \in \mathbb{N}_{0}\right)
$$

were derived in the recent works (Bin-Saad 2007), (Gupta and Kumari 2011) and (Saxena et al. (2011a)).

Remark 7. Two of the claimed integral formulas in BinSaad's paper (2007, p. 42, Theorem 3.2, Equations (3.10) and (3.11)) can easily be shown to be divergent, simply because the improper integrals occurring on their lefthand sides obviously violate the required convergence conditions at their lower terminal $t=0$.

We now turn toward the truncated forms of the generating functions $\Omega_{\lambda}(z, t ; s, a)$ and $\Theta(z, t ; s, a)$ in (3.2) and (3.3), respectively, which are defined by (3.4) to (3.7). Indeed, by appealing appropriately to the definitions in (3.4) to (3.7) in conjunction with the Eulerian Gamma-function integral in (3.13), it is fairly straightforward to derive the integral representation formulas asserted by Theorem 5 below.

Theorem 5. In terms of the sequence $\left\{\Xi_{n}\right\}_{n \in \mathbb{N}_{0}}$ of the coefficients given by the definition (2.13), each of the following Eulerian Gamma-function integral formulas holds true:

$$
\begin{aligned}
& \Omega_{\lambda}^{(0, r)}(z, \omega ; s, a) \\
& =\frac{1}{\Gamma(s)} \int_{0}^{\infty} t^{s-1} \mathrm{e}^{-a t}\left(\sum_{k=0}^{r} \Xi_{k}\left(z \mathrm{e}^{-t}\right)^{k}\right){ }_{1} F_{1}(\lambda ; s ; \omega t) \mathrm{d} t \\
& \quad(\min \{\Re(s), \Re(a)\}>0), \\
& \Omega_{\lambda}^{(r+1, \infty)}(z, \omega ; s, a) \\
& =\frac{1}{\Gamma(s)} \int_{0}^{\infty} t^{s-1} \mathrm{e}^{-a t}\left(\sum_{k=r+1}^{\infty} \Xi_{k}\left(z \mathrm{e}^{-t}\right)^{k}\right){ }_{1} F_{1}(\lambda ; s ; \omega t) \mathrm{d} t \\
& \quad(\min \{\Re(s), \Re(a)\}>0),
\end{aligned}
$$

$$
\begin{aligned}
& \Theta^{(0, \mathrm{r})}(z, \omega ; s, a) \\
& =\frac{1}{\Gamma(s)} \int_{0}^{\infty} t^{s-1} \mathrm{e}^{-a t}\left(\sum_{k=0}^{r} \Xi_{k}\left(z \mathrm{e}^{-t}\right)^{k}\right){ }_{0} F_{1}(\longleftarrow ; s ; \omega t) \mathrm{d} t \\
& \quad(\min \{\Re(s), \Re(a)\}>0)
\end{aligned}
$$

and

$$
\begin{aligned}
& \Theta^{(\mathrm{r}+1, \infty)}(z, \omega ; s, a) \\
& =\frac{1}{\Gamma(s)} \int_{0}^{\infty} t^{s-1} \mathrm{e}^{-a t}\left(\sum_{k=r+1}^{\infty} \Xi_{k}\left(z \mathrm{e}^{-t}\right)^{k}\right){ }_{0} F_{1}(-; s ; \omega t) \mathrm{d} t \\
& \quad(\min \{\Re(s), \Re(a)\}>0),
\end{aligned}
$$


provided that both sides of each of the assertions (3.26) to (3.29) exist.

Remark 8. Several specialized cases of Theorem 5 when

$$
\Xi_{n}=1 \quad\left(n \in \mathbb{N}_{0}\right)
$$

can be found in the recent works (Bin-Saad 2007), (Gupta and Kumari 2011) and (Saxena et al. (2011a)).

It is not difficult to derive various other properties and results involving the generating functions $\Omega_{\lambda}(z, t ; s, a)$ and $\Theta(z, t ; s, a)$ in (3.2) and (3.3), respectively, as well as their truncated forms which are defined by (3.4) to (3.7). For example, by applying the definition (3.2) in conjunction with the definition (2.15), it is easy to derive the following general form of the generating relations asserted by (for example) Bin-Saad (2007, p. 44, Theorem 4.2):

$$
\begin{aligned}
& \sum_{n=0}^{\infty} \frac{\left(\alpha_{1}\right)_{n u_{1}} \cdots\left(\alpha_{\ell}\right)_{n u_{\ell}}}{\left(\beta_{1}\right)_{n v_{1}} \cdots\left(\beta_{m}\right)_{n v_{m}}} \Omega_{\lambda}(z, \omega ; s+n, a) \frac{t^{n}}{n !} \\
& =\sum_{k=0}^{\infty} \Xi_{k}\left(1-\frac{\omega}{k+a}\right)^{-\lambda} \\
& \cdot \ell \Psi_{m}^{*}\left[\begin{array}{c}
\left(\alpha_{1}, u_{1}\right), \cdots,\left(\alpha_{\ell}, u_{\ell}\right) ; \frac{t}{k+a} \\
\left(\beta_{1}, v_{1}\right), \cdots,\left(\beta_{m}, v_{m}\right) ; \frac{z^{k}}{(k+a)^{s}}
\end{array}\right. \\
& \left(\ell, m \in \mathbb{N}_{0} ; \alpha_{j} \in \mathbb{C}, u_{j} \in \mathbb{R}^{+}(j=1, \cdots, \ell) ;\right. \\
& \left.\beta_{j} \in \mathbb{C} \backslash \mathbb{Z}_{0}^{-}, v_{j} \in \mathbb{R}^{+}(j=1, \cdots, m) ; \max \{|\omega|,|t|\}<1\right),
\end{aligned}
$$

where the sequence $\left\{\Xi_{n}\right\}_{n \in \mathbb{N}_{0}}$ of the coefficients is given by the definition (2.13) and it is tacitly assumed that each member of the generating relation (3.30) exists. We do, however, choose to leave the details involved in all such derivations as exercises for the interested reader.

\section{$\tau$-Generalizations of the Hurwitz-Lerch Zeta functions}

In a recent paper, Saxena et al. (2011a) considered a socalled $\tau$-generalization of the Hurwitz-Lerch Zeta function $\Phi(z, s, a)$ in (1.1) in the following form [Saxena et al. ((2011a), p. 311, Equation (2.1))]:

$$
\Phi(\tau ; z, s, a):=\sum_{n=0}^{\infty} \frac{z^{n}}{(\tau n+a)^{s}} \quad\left(\tau \in \mathbb{R}^{+}\right) .
$$

Subsequently, by similarly introducing a parameter $\tau>0$ in the definition (2.5), Gupta and Kumari (2011) studied a $\tau$-generalization of the extended Hurwitz-Lerch Zeta function $\Phi_{\mu}^{*}(z, s, a)$ in (2.5) as follows:

$$
\Phi_{\mu}^{*}(\tau ; z, s, a):=\sum_{n=0}^{\infty} \frac{(\mu)_{n}}{n !} \frac{z^{n}}{(\tau n+a)^{s}} \quad\left(\tau \in \mathbb{R}^{+}\right),
$$

which, when compared with the definition (4.1), yields the relationship:

$$
\Phi(\tau ; z, s, a)=\Phi_{1}^{*}(\tau ; z, s, a) \quad\left(\tau \in \mathbb{R}^{+}\right) .
$$

By looking closely at the definitions (4.1) and (4.2), in conjunction with the earlier definitions (1.1) and (2.5), respectively, we immediately get the following rather obvious connections:

$$
\begin{aligned}
\Phi(\tau ; z, s, a) & =\frac{1}{\tau^{s}} \Phi\left(z, s, \frac{a}{\tau}\right) & & \text { or } \\
\Phi(z, s, a) & =\tau^{s} \Phi(\tau ; z, s, a \tau) & & \left(\tau \in \mathbb{R}^{+}\right)
\end{aligned}
$$

and

$$
\begin{array}{rlrl}
\Phi_{\mu}^{*}(\tau ; z, s, a) & =\frac{1}{\tau^{s}} \Phi_{\mu}^{*}\left(z, s, \frac{a}{\tau}\right) & & \text { or } \\
\Phi_{\mu}^{*}(z, s, a) & =\tau^{s} \Phi_{\mu}^{*}(\tau ; z, s, a \tau) & \left(\tau \in \mathbb{R}^{+}\right)
\end{array}
$$

Clearly, therefore, the definitions in (4.1) and (4.2) (with $\tau \in \mathbb{R}^{+}$) are no more general than their corresponding well-known cases when $\tau=1$ given by the definitions in (1.1) and (2.5), respectively. Thus, by trivially appealing to the parametric changes exhibited by the connections in (4.4) and (4.5), all of the results involving the so-called $\tau$ generalized functions $\Phi(\tau ; z, s, a)$ and $\Phi_{\mu}^{*}(\tau ; z, s, a)$ can be derived simply from the corresponding (usually known) results involving the familiar functions $\Phi(z, s, a)$ and $\Phi_{\mu}^{*}(z, s, a)$, respectively. Just for illustration of the triviality associated with such straightforward derivations, we recall the following sum-integral representation formula due to Lin and Srivastava ((2004), p. 729, Equation (20)) (see also Srivastava et al. ((2011), p. 494, Equation (2.6)) for the special case when $k=1)$ :

$$
\begin{aligned}
& \Phi_{\mu, \nu}^{(\rho, \sigma)}(z, s, a) \\
& =\frac{1}{\Gamma(s)} \sum_{j=0}^{k-1} \frac{(\mu)_{\rho j}}{(v)_{\sigma j}} z^{j} \\
& \quad \cdot \int_{0}^{\infty} t^{s-1} \mathrm{e}^{-(a+j) t}{ }_{2} \Psi_{1}\left[\begin{array}{c}
(\mu+\rho j, \rho k),(1,1) ; \\
(v+\sigma j, \sigma k) ;
\end{array} z^{k} \mathrm{e}^{-k t}\right] \mathrm{d} t \\
& (k \in \mathbb{N} ; \min \{\Re(a), \Re(s)\}>0 ; \sigma>\rho>0 \quad \text { when } \\
& \left.z \in \mathbb{C} ; \sigma \geqq \rho>0 \quad \text { when } \quad|z|^{1 / k}<\rho^{-\rho} \sigma^{\sigma}\right),
\end{aligned}
$$

it being tacitly assumed that each member of (4.6) exists. Indeed, in the special case when $\rho=\sigma=v=1$, (4.6) yields the following sum-integral representation for 
the generalized Hurwitz-Lerch Zeta function $\Phi_{\mu}^{*}(z, s, a)$ involved in (2.5):

$$
\begin{aligned}
& \Phi_{\mu}^{*}(z, s, a) \\
& =\frac{1}{\Gamma(s)} \sum_{j=0}^{k-1} \frac{(\mu)_{j}}{(v)_{j}} z^{j} \\
& \quad \cdot \int_{0}^{\infty} t^{s-1} \mathrm{e}^{-(a+j) t}{ }_{2} \Psi_{1}\left[\begin{array}{c}
(\mu+j, k),(1,1) ; \\
(v+j, k) ; \\
\left.z^{k} \mathrm{e}^{-k t}\right] \mathrm{d} t
\end{array}\right] \\
& (k \in \mathbb{N} ; \min \{\Re(a), \Re(s)\}>0 ;|z|<1)
\end{aligned}
$$

or, equivalently,

$$
\begin{aligned}
& \Phi_{\mu}^{*}(z, s, a) \\
& =\frac{1}{\Gamma(s)} \sum_{j=0}^{k-1} \frac{(\mu)_{j}}{(v)_{j}} z^{j} \\
& \quad \cdot \int_{0}^{\infty} t^{s-1} \mathrm{e}^{-(a+j) t}{ }_{k+1} F_{k}\left[\begin{array}{c}
\Delta^{*}(k ; \mu+j),(1,1) ; \\
z^{k} \mathrm{e}^{-k t}
\end{array}\right] \mathrm{d} t
\end{aligned}
$$$$
(k \in \mathbb{N} ; \min \{\Re(a), \Re(s)\}>0 ;|z|<1),
$$

where, for convenience, $\Delta^{*}(n ; \lambda)$ abbreviates the array of $n$ parameters

$$
\frac{\lambda}{n}, \frac{\lambda+1}{n}, \cdots, \frac{\lambda+n-1}{n} \quad(n \in \mathbb{N}),
$$

the array being empty when $n=0$.

Now, in order to rewrite this last result (4.8) in terms of the $\tau$-generalized Hurwitz-Lerch Zeta function $\Phi_{\mu}^{*}(\tau ; z, s, a)$ defined by (4.2), we simply make the following parameter and variable changes:

$$
a \mapsto \frac{a}{\tau}, t \mapsto \tau t \quad \text { and } \quad \mathrm{d} t \mapsto \tau \mathrm{d} t \quad\left(\tau \in \mathbb{R}^{+}\right)
$$

and multiply the resulting equation by $\tau^{-s}$. By using the connection in (4.5), we thus find immediately that

$$
\begin{aligned}
& \Phi_{\mu}^{*}(\tau ; z, s, a) \\
& =\frac{1}{\Gamma(s)} \sum_{j=0}^{k-1} \frac{(\mu)_{j}}{(v)_{j}} z^{j} \\
& \quad \cdot \int_{0}^{\infty} t^{s-1} \mathrm{e}^{-(a+\tau j) t}{ }_{k+1} F_{k}\left[\begin{array}{c}
\Delta^{*}(k ; \mu+j),(1,1) ; \\
\Delta^{*}(k ; v+j) ;
\end{array} \mathrm{e}^{-k \tau t}\right] \mathrm{d} t
\end{aligned}
$$

$(k \in \mathbb{N} ; \min \{\Re(a), \Re(s)\}>0 ;|z|<1)$.
In its particular case when $k=1$, this last formula (4.9) would simplify at once to the following form given by Saxena et al. ((2011a), p. 311, Equation (2.2)):

$$
\begin{aligned}
& \Phi_{\mu}^{*}(\tau ; z, s, a)=\frac{1}{\Gamma(s)} \int_{0}^{\infty} t^{s-1} \mathrm{e}^{-a t}\left(1-z \mathrm{e}^{-\tau t}\right)^{-\mu} \mathrm{d} t \\
& (\Re(a)>0 ; \Re(s)\}>0 \quad \text { when } \quad|z|<1 ; \Re(s)>1 \\
& \text { when } z=1),
\end{aligned}
$$

which obviously is equivalent to (and certainly not a generalization of) of the $\tau=1$ case derived earlier by Goyal and Laddha ((1997), p. 100, Equation (1.6)).

Remark 9. The so-called $\tau$-generalizations ${ }_{2} R_{1}^{\tau}$ and ${ }_{1} R_{1}^{\tau}$ of the Gauss hypergeometric function ${ }_{2} F_{1}$ and Kummer's confluent hypergeometric function ${ }_{1} F_{1}$, respectively, which were used in the aforecited paper by Saxena et al. ((2011a), p. 315), are obviously very specialized cases of the well-known and extensively-investigated Fox-Wright function $p \Psi_{q}$ defined by (2.15). In fact, it is easily seen from Definition 2 that [Saxena et al. ((2011a), pp. 315 and 317)] (see also (Al-Zamel 2001), (Ali et al. 2001) and (Virchenko et al. 2001))

$$
\begin{aligned}
{ }_{2} R_{1}^{\tau}(a, b ; c ; z) & :=\frac{\Gamma(c)}{\Gamma(b)} \sum_{n=0}^{\infty} \frac{(a)_{n} \Gamma(b+\tau n)}{\Gamma(c+\tau n)} \frac{z^{n}}{n !} \\
& =\frac{\Gamma(c)}{\Gamma(a) \Gamma(b)}{ }_{2} \Psi_{1}\left[\begin{array}{r}
(a, 1),(b, \tau) ; \\
z \\
(c, \tau) ;
\end{array}\right] \\
& ={ }_{2} \Psi_{1}^{*}\left[\begin{array}{c}
(a, 1),(b, \tau) ; \\
z \\
(c, \tau) ;
\end{array}\right] \\
& \left(|z|<1 ; \tau \in \mathbb{R}^{+} ; c \notin \mathbb{Z}_{0}^{-}\right)
\end{aligned}
$$

and

$$
\begin{aligned}
{ }_{1} R_{1}^{\tau}(b ; c ; z): & =\frac{\Gamma(c)}{\Gamma(b)} \sum_{n=0}^{\infty} \frac{\Gamma(b+\tau n)}{\Gamma(c+\tau n)} \frac{z^{n}}{n !} \\
& =\frac{\Gamma(c)}{\Gamma(b)}{ }_{1} \Psi_{1}\left[\begin{array}{c}
(b, \tau) ; \\
z \\
(c, \tau) ;
\end{array}\right] \\
& ={ }_{1} \Psi_{1}^{*}\left[\begin{array}{c}
(b, \tau) ; \\
z \\
(c, \tau) ;
\end{array}\right] \\
& \left(|z|<\infty ; \tau \in \mathbb{R}^{+} ; c \notin \mathbb{Z}_{0}^{-}\right) .
\end{aligned}
$$

Similar remarks and observations would apply equally strongly to the other $\tau$-generalizations of well-known and extensively-investigated hypergeometric functions in one, two and more variables. 
We conclude this section by presenting a generalization of the sum-integral representation formula (4.6) due to Lin and Srivastava ((2004), p. 729, Equation (20)).

Theorem 6. The following sum-integral representation formula holds true:

$$
\begin{gathered}
\Phi_{\lambda_{1}, \cdots, \lambda_{p} ; \mu_{1}, \cdots, \mu_{q}}^{\left(\rho_{1}, \cdots, \rho_{p}, \sigma_{1}, \cdots, \sigma_{q}\right)}(z, s, a)=\frac{1}{\Gamma(s)} \sum_{j=0}^{k-1} \frac{\prod_{\ell=1}^{p}\left(\lambda_{\ell}\right)_{j \rho_{\ell}}}{\prod_{\ell=1}^{q}\left(\mu_{\ell}\right)_{j \sigma_{\ell}}} \frac{z^{j}}{j !} \int_{0}^{\infty} t^{s-1} \mathrm{e}^{-(a+j) t} \\
\cdot p+1 \Psi_{q+1}^{*}\left[\begin{array}{c}
\left(\lambda_{1}+j \rho_{1}, k \rho_{1}\right), \cdots,\left(\lambda_{p}+j \rho_{p}, k \rho_{p}\right),(1,1) ; \\
\left(\mu_{1}+j \sigma_{1}, k \sigma_{1}\right), \cdots,\left(\mu_{q}+j \sigma_{q}, k \sigma_{q}\right),(j+1, k) ;
\end{array} z^{k} \mathrm{e}^{-k t}\right] \mathrm{d} t
\end{gathered}
$$

$(k \in \mathbb{N} ; \min \{\Re(a), \mathfrak{R}(s)\}>0)$,

provided that each member of the assertion (4.13) exists.

Proof. First of all, in light of the following elementary series identity:

$$
\sum_{n=0}^{\infty} f(n)=\sum_{j=0}^{k-1} \sum_{n=0}^{\infty} f(k n+j) \quad(k \in \mathbb{N})
$$

we find from the definition (2.12) that

$$
\begin{gathered}
\Phi_{\lambda_{1}, \cdots, \lambda_{p} ; \mu_{1}, \cdots, \mu_{q}}^{\left(\rho_{1}, \cdots, \rho_{p}, \sigma_{1}, \cdots, \sigma_{q}\right)}(z, s, a)=k^{-s} \sum_{j=0}^{k-1} \frac{\prod_{\ell=1}^{p}(\lambda)_{j \rho_{\ell}}}{\prod_{\ell=1}^{q}\left(\mu_{\ell}\right)_{j \sigma_{\ell}}} \frac{z^{j}}{j !} \\
\cdot \Phi_{\lambda_{1}+j \rho_{1}, \cdots, \lambda p+j \rho_{p}, 1 ; \mu_{1}+j \sigma_{1}, \cdots, \mu_{q}+j \sigma_{q}, j+1}^{\left(k \rho_{1}, \cdots, k \rho_{p}, 1, k \sigma_{1}, \cdots, k \sigma_{q}, k\right)} \\
\left(z^{k}, s, \frac{a+j}{k}\right)
\end{gathered}
$$

The assertion (4.13) of Theorem 6 would now emerge readily upon first appealing to the aforementioned known result due to Srivastava et al. ((2011), p. 504, Equation (6.4)) (see also Remark 3 above) given by

$$
\begin{aligned}
& \Phi_{\lambda_{1}, \cdots, \lambda_{p} ; \mu_{1}, \cdots, \mu_{q}}^{\left(\rho_{1}, \cdots, \rho_{p}, \sigma_{1}, \cdots, \sigma_{q}\right)}(z, s, a) \\
& \left.\quad=\frac{1}{\Gamma(s)} \int_{0}^{\infty} t^{s-1} \mathrm{e}^{-a t}{ }_{p} \Psi_{q}^{*}\left[\begin{array}{c}
\left(\lambda_{1}, \rho_{1}\right), \cdots,\left(\lambda_{p}, \rho_{p}\right) ; \\
\left(\mu_{1}, \sigma_{1}\right), \cdots,\left(\mu_{q}, \sigma_{q}\right) ;
\end{array}\right] \mathrm{e} \mathrm{e}^{-t}\right] \mathrm{d} t \\
& (\min \{\Re(a), \Re(s)\}>0)
\end{aligned}
$$

and then setting

$$
t \mapsto k t \quad \text { and } \quad \mathrm{d} t \mapsto k \mathrm{~d} t \quad(k \in \mathbb{N})
$$

Obviously, in its special case when

$p=2 \quad\left(\lambda_{1}=\mu \quad\right.$ and $\quad \rho_{1}=\rho ; \lambda_{2}=1 \quad$ and $\left.\quad \rho_{2}=1\right)$

and

$$
q=1 \quad\left(\mu_{1}=v \text { and } \sigma_{1}=\sigma\right),
$$

the general result (4.13) asserted by Theorem 6 would reduce immediately to the known sum-integral representation formula (4.6) due to Lin and Srivastava ((2004), p. 729, Equation (20)).

\section{Competing interests}

The author declares that they have no competing interests.

\section{Acknowledgements}

The present investigation was supported, in part, by the Natural Sciences and Engineering Research Council of Canada under Grant OGP0007353.

Received: 20 November 2012 Accepted: 4 January 2013

Published: 25 February 2013

\section{References}

Al-Zamel A (2001) On a generalized gamma-type distribution with $\tau$-confluent hypergeometric function. Kuwait J Sci Engrg 28: 25-36

Ali I, Kalla SL, Khajah HG (2001) A generalized inverse Gaussian distribution with $\tau$-confluent hypergeometric function. Integral Transforms Spec Funct 12: $101-114$

Bin-Saad MG (2007) Sums and partial sums of double power series associated with the generalized Zeta function and their $N$-Fractional calculus. Math J Okayama Univ 49: 37-52

Buschman RG, Srivastava HM (1990) The $\bar{H}$-function associated with a certain class of Feynman integrals. J Phys A: Math Gen 23: 4707-4710

Choi J, Jang DS, Srivastava HM (2008) A generalization of the Hurwitz-Lerch Zeta function. Integral Transforms Spec Funct 19: 65-79

Erdélyi A, Magnus W, Oberhettinger F, Tricomi FG (1953) Higher Transcendental Functions, Vol. I. McGraw-Hill Book Company, New York, Toronto and London

Erdélyi, A, Magnus W, Oberhettinger F, Tricomi FG (1954) Tables of Integral Transforms, Vol. II. McGraw-Hill Book Company, New York, Toronto and London

Garg M, Jain K, Kalla SL (2008) A further study of general Hurwitz-Lerch Zeta function. Algebras Groups Geom. 25: 311-319

Garg M, Jain K, Srivastava HM (2006) Some relationships between the generalized Apostol-Bernoulli polynomials and Hurwitz-Lerch Zeta functions. Integral Transforms Spec Funct 17: 803-815 
Goyal SP, Laddha RK (1997) On the generalized Zeta function and the generalized Lambert function. Ganita Sandesh 11: 99-108

Gupta RK, Kumari M (2011) Some results on a $\tau$-generalized Riemann Zeta function. Jñānābha 41: 63-68

Gupta PL, Gupta RC, Ong S-H, Srivastava HM (2008) A class of Hurwitz-Lerch Zeta distributions and their applications in reliability. Appl Math Comput 196: 521-531

Inayat-Hussain AA (1987a) New properties of hypergeometric series derivable from Feynman integrals. I: Transformation and reduction formulae. J Phys A: Math Gen 20: 4109-4117

Inayat-Hussain AA (1987b) New properties of hypergeometric series derivable from Feynman integrals. II: A generalization of the $H$-function. J Phys A: Math Gen 20: 4119-4128

Jankov D, Pogány TK, Saxena RK (2011) An extended general Hurwitz-Lerch Zeta function as a Mathieu $(\boldsymbol{a}, \boldsymbol{\lambda})$-series. Appl Math Lett 24: 1473-1476

Katsurada M (1997) On Mellin-Barnes type of integrals and sums associated with the Riemann Zeta-function. Publ Inst Math (Beograd) (Nouvelle Ser) 62(76): 13-25

Kilbas AA, Saigo M, Trujillo JJ (2002) On the generalized Wright functions. Fract Calc Appl Anal 5: 437-466

Kilbas AA, Srivastava HM, Trujillo JJ (2006) Theory and Applications of Fractional Differential Equations, North-Holland Mathematical Studies, Vol. 204. Elsevier (North-Holland) Science Publishers, Amsterdam, London and New York

Lin S-D, Srivastava HM (2004) Some families of the Hurwitz-Lerch Zeta functions and associated fractional derivative and other integral representations. Appl Math Comput 154: 725-733

Lin S-D, Srivastava HM, Wang P-Y (2006) Some expansion formulas for a class of generalized Hurwitz-Lerch Zeta functions. Integral Transforms Spec Funct 17: 817-827

Mathai AM, Saxena RK, Haubold HJ (2010) The $H$-Function: Theory and Applications. Springer, New York, Dordrecht, Heidelberg and London

Nishimoto K, Yen C-E, Lin M-L (2002) Some integral forms for a generalized Zeta function. J Fract Calc 22: 91-97

Răducanu D, Srivastava HM (2007) A new class of analytic functions defined by means of a convolution operator involving the Hurwitz-Lerch Zeta function. Integral Transforms Spec Funct 18: 933-943

Samko SG, Kilbas AA, Marichev OI (1993) Fractional Integrals and Derivatives: Theory and Applications, Translated from the Russian: Integrals and Derivatives of Fractional Order and Some of Their Applications ("Nauka i Tekhnika", Minsk, 1987). Gordon and Breach Science Publishers, Reading, Tokyo, Paris, Berlin and Langhorne (Pennsylvania)

Saxana RK (2009) A remark on a paper on $M$-series [MR2401326 (2009b:26008)]. With an editorial note. Fract Calc Appl Anal 12: 109-110

Saxena RK, Gupta RK, Kumari M (2011a) Integrals and series expansions of the $\tau$-generalized Riemann Zeta function. J Indian Acad Math 33: 309-320

Saxena RK, Pog'any TK, Saxena R, Jankov D (2011 b) On generalized Hurwitz-Lerch Zeta distributions occurring in statistical inference. Acta Univ Sapientiae Math 3: 43-59

Sharma M (2008) Fractional integration and fractional differentiation of the $M$-series. Fract Calc Appl Anal 11:187-191

Sharma M, Jain R (2009) A note on a generalzed $M$-series as a special function of fractional calculus. Fract Calc Appl Anal 12: 449-452

Srivastava HM (1988a) Sums of certain series of the Riemann Zeta function J Math Anal Appl 134: 129-140

Srivastava, H M (1988b) A unified presentation of certain classes of series of the Riemann Zeta function. Riv Mat Univ Parma (Ser. 4) 14: 1-23

Srivastava HM (2000) Some formulas for the Bernoulli and Euler polynomials at rational arguments. Math Proc Cambridge Philos Soc 129: 77-84

Srivastava, H M (2011) Some generalizations and basic (or $q$-) extensions of the Bernoulli, Euler and Genocchi polynomials. Appl Math Inform Sci 5: $390-444$

Srivastava HM, Choi J (2001) Series Associated with the Zeta and Related Functions. Kluwer Academic Publishers, Dordrecht, Boston and London

Srivastava HM, Choi J (2012) Zeta and $q$-Zeta Functions and Associated Series and Integrals. Elsevier Science Publishers, Amsterdam, London and New York

Srivastava HM, Garg M, Choudhary S (2010) A new generalization of the Bernoulli and related polynomials. Russian J Math Phys 17: 251-261

Srivastava HM, Gupta KC, Goyal SP (1982) The $H$-Functions of One and Two Variables with Applications. South Asian Publishers, New Delhi and Madras
Srivastava HM, Jankov D, Pogány TK, Saxena RK (2011) Two-sided inequalities for the extended Hurwitz-Lerch Zeta function. Comput Math Appl 62: 516-522

Srivastava HM, Karlsson PW (1985) Multiple Gaussian Hypergeometric Series, Halsted Press (Ellis Horwood Limited, Chichester). John Wiley and Sons, New York, Chichester, Brisbane and Toronto

Srivastava HM, Lin S-D, Wang P-Y (2006) Some fractional-calculus results for the $\bar{H}$-function associated with a class of Feynman integrals. Russian J Math Phys 13: 94-100

Srivastava HM, Manocha HL (1984) A Treatise on Generating Functions, Halsted Press (Ellis Horwood Limited, Chichester). John Wiley and Sons, New York, Chichester, Brisbane and Toronto

Srivastava HM, Saxena RK, Pogány TK, Saxena R (2011) Integral and computational representations of the extended Hurwitz-Lerch Zeta function. Integral Transforms Spec Funct 22: 487-506

Virchenko NO, Kalla SL, Al-Zamel A (2001) Some results on a generalized hypergeometric function. Integral Transforms Spec Funct 12: 89-100

Wilton JR (1922/1923) A proof of Burnside's formula for $\log \Gamma(x+1)$ and certain allied properties of Riemann's $\zeta$-function. Messenger Math 52:90-93

Yen C-E, Lin M-L, Nishimoto K (2002) An integral form for a generalized Zeta function. J Fract Calc 23: 99-102

\section{doi:10.1186/2193-1801-2-67}

Cite this article as: Srivastava: Generating relations and other results associated with some families of the extended Hurwitz-Lerch Zeta functions. SpringerPlus 2013 2:67.

\section{Submit your manuscript to a SpringerOpen ${ }^{\mathcal{O}}$ journal and benefit from:}

- Convenient online submission

- Rigorous peer review

- Immediate publication on acceptance

- Open access: articles freely available online

- High visibility within the field

- Retaining the copyright to your article

Submit your next manuscript at $\boldsymbol{\wedge}$ springeropen.com 